OPEN ACCESS

Edited by:

Mario Malerba,

Università del Piemonte Orientale, Italy

Reviewed by:

Stefania Loffredo,

University of Naples Federico II, Italy

Kjell Alving,

Uppsala University, Sweden

*Correspondence:

Corrado Pelaia

pelaia.corrado@gmail.com

Specialty section:

This article was submitted to Respiratory Pharmacology,

a section of the journal

Frontiers in Pharmacology

Received: 11 January 2022

Accepted: 21 February 2022

Published: 08 March 2022

Citation:

Pelaia C, Heffler E, Crimi C, Maglio A, Vatrella A, Pelaia G and Canonica GW (2022) Interleukins 4 and 13 in Asthma: Key Pathophysiologic Cytokines and

Druggable Molecular Targets.

Front. Pharmacol. 13:851940.

doi: 10.3389/fphar.2022.851940

\section{Interleukins 4 and 13 in Asthma: Key Pathophysiologic Cytokines and Druggable Molecular Targets}

\author{
Corrado Pelaia ${ }^{1 *}$, Enrico Heffler ${ }^{2,3}$, Claudia Crimi ${ }^{4}$, Angelantonio Maglio ${ }^{5}$, \\ Alessandro Vatrella ${ }^{5}$, Girolamo Pelaia ${ }^{1}$ and Giorgio Walter Canonica ${ }^{2,3}$ \\ ${ }^{1}$ Department of Health Sciences, University "Magna Græcia" of Catanzaro, Catanzaro, Italy, ${ }^{2}$ Personalized Medicine, Asthma and \\ Allergy, Humanitas Clinical and Research Center IRCCS, Rozzano, Italy, ${ }^{3}$ Department of Biomedical Sciences, Humanitas \\ University, Pieve Emanuele, Italy, ${ }^{4}$ Department of Clinical and Experimental Medicine, University of Catania, Catania, Italy, \\ ${ }^{5}$ Department of Medicine, Surgery, and Dentistry, University of Salerno, Salerno, Italy
}

Interleukins (IL)-4 and -13 play a pivotal role in the pathobiology of type-2 asthma. Indeed, IL-4 is crucially involved in Th2 cell differentiation, immunoglobulin (lg) class switching and eosinophil trafficking. IL-13 cooperates with IL-4 in promoting IgE synthesis, and also induces nitric oxide (NO) production, goblet cell metaplasia and fibroblast proliferation, as well as elicits contractile responses and hyperplasia of airway smooth muscle cells.

$\mathrm{IL}-4$ and IL-13 share common signaling pathways, activated by the binding of both cytokines to receptor complexes including the $\alpha$-subunit of the IL-4 receptor (IL-4Ra). Therefore, the subsequent receptor dimerization is responsible for the pathophysiologic effects of IL-4 and IL-13. By selectively blocking IL-4Ra, the fully human IgG4 monoclonal antibody dupilumab behaves as a dual receptor antagonist of both IL-4 and IL-13. Through this mechanism of action, dupilumab exerts effective therapeutic actions in type-2 inflammation, thus decreasing asthma exacerbations, FeNO (fractional exhaled NO) levels, and the intake of oral corticosteroids (OCS). In addition to being approved for the add-on biological therapy of severe asthma, dupilumab has also been licensed for the treatment of nasal polyposis and atopic dermatitis.

Keywords: severe asthma, IL-4, IL-13, dupilumab, IL-4 receptor

\section{INTRODUCTION}

Asthma is a widespread respiratory disorder, usually characterized by variable airflow limitation associated with airway inflammation and remodeling (Holgate et al., 2015; Khalaf et al., 2019; Stern et al., 2020). This heterogeneous disease includes several phenotypes, originating from complex interactions between genetic, and environmental factors (Kuruvilla et al., 2019; Thomsen, 2015). The different asthmatic phenotypes are mainly shaped by distinct inflammatory profiles, underpinned by intricate networks of cellular and molecular pathomechanisms known as endotypes, which can also be responsible for the development of severe clinical features (Komlosi et al., 2022; Wenzel, 2021). In particular, the multiple variants of asthma may consist of either eosinophilic, neutrophilic, mixed, or paucigranulocytic patterns (Papi et al., 2018; Suraya et al., 2021; Carr et al., 2018; Tliba and Panettieri, 2019). Eosinophilic airway infiltration is the dominant effector inflammatory trait of type-2 (T2) allergic or non-allergic asthma, driven by interacting innate and adaptive immune responses orchestrated by group 2 innate lymphoid cells (ILC2) and T helper 2 (Th2) lymphocytes, producing the interleukins 4 (IL- 

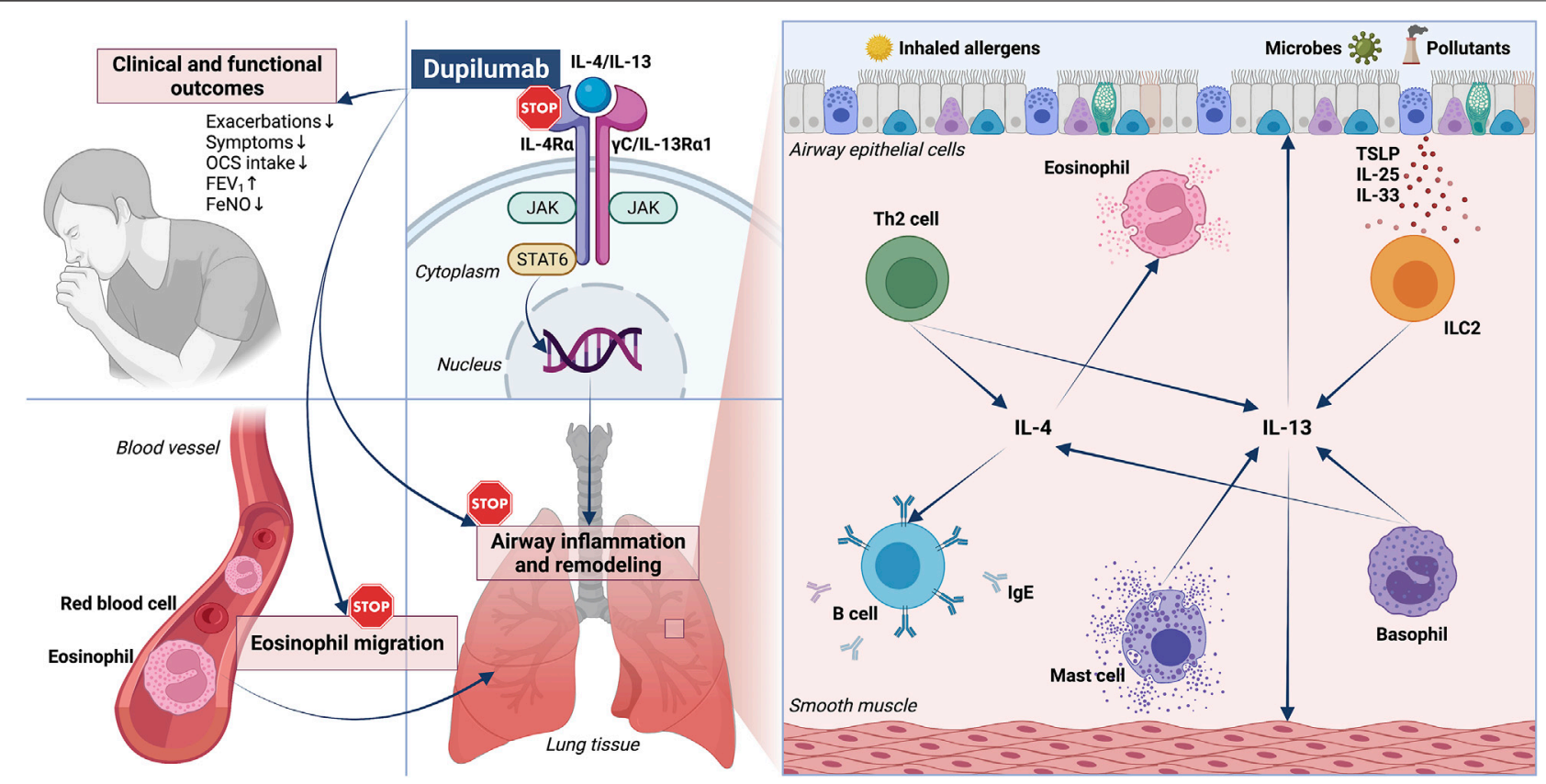

FIGURE 1 | Pathophysiology and biological therapy of type 2 asthma: respective roles of IL-4/IL-13 and dual receptor blockade by dupilumab. RIGHT PANEL-A key role in the pathobiology of type 2 asthma is played by both IL-4 and IL-13, released by Th2 lymphocytes, ILC2, basophils, and mast cells. IL-4 and IL-13 act on many cellular targets, including immune inflammatory cells such as B lymphocytes and eosinophils, as well as airway structural cells (epithelial cells and smooth muscle cells). LEFT PANEL-By binding to IL-4Ra, dupilumab operates a receptor blockade which suppresses the biological effects of IL-4 and IL-13, and is thus responsible for several therapeutic effects including clinical and functional improvements, as well as inhibition of eosinophil trafficking. IL-4Ra: $\alpha$ subunit of IL-4 receptor; IL-13R 1 : $\alpha 1$ subunit of IL-13 receptor; JAK: Janus kinase; STAT: signal transducer and activator of transcription; TSLP: thymic stromal lymphopoietin; ILC2: group 2 innate lymphoid cells.

4), 13 (IL-13), and 5 (IL-5) (Nelson et al., 2020; RodriguezRodriguez et al., 2021; Hammad and Lambrecht, 2021). In type2 asthma, these cytokines exert key functions with regard to inception, persistence and amplification of bronchial inflammation and remodeling. Indeed, IL-4 promotes both Th2 cell differentiation and biosynthesis of immunoglobulins $\mathrm{E}$ (IgE), whereas IL-13 is mostly responsible for airway hyperresponsiveness, mucus overproduction, and bronchial structural changes (Figure 1) (Matucci et al., 2021). Moreover, IL-5 is the principal inducer of maturation, survival, proliferation, and activation of eosinophils (Pelaia et al., 2019). Within such a pathogenic context, a crucial role is played by tissue damage occurring at level of the bronchial epithelium, which releases copious amounts of alarmins when injured by one or more of many triggers including allergens, cigarette smoke, airborne pollutants, and infectious agents (Calvén et al., 2020). Alarmins comprehend the innate cytokines thymic stromal lymphopoietin (TSLP), interleukin25 (IL-25) and interleukin-33 (IL-33), which act as upstream stimulators of both innate, and adaptive immune cascades implicated in type-2 asthma (Hong et al., 2020). In fact, alarmins directly activate ILC2, and also prime conventional dendritic cells to elicit the maturation and clonal expansion of Th2 lymphocytes (Hammad and Lambrecht, 2021; Komlosi et al., 2022). As a consequence, alarmins induce the release of type- 2 cytokines such as IL-13, which in turn enhances airway epithelial cell secretion of IL-33, thereby nurturing a feed- forward pathogenic circuit that significantly amplifies type-2 inflammation in asthmatic patients (Christianson et al., 2015).

The above arguments explain why IgE, IL-4/IL-13 receptors, IL-5 and its receptor, as well as alarmins and their receptors represent suitable molecular targets for current and prospective biological therapies of severe asthma (Pelaia et al., 2020; Pelaia et al., 2022; Busse et al., 2021; Porsbjerg et al., 2020; Albrecht, 2021). In particular, the fully human monoclonal antibody dupilumab behaves as a dual IL-4/IL-13 receptor antagonist, which according to both randomized trials and real-life studies has been shown to effectively inhibit type- 2 inflammation, thus significantly improving several clinical and functional parameters related to asthma (Figure 1), nasal polyposis, and atopic dermatitis (Pelaia et al., 2017; Ricciardolo et al., 2021).

Therefore, the present narrative review aims to provide an updated coverage of the following two topics: 1) pathobiologic roles of IL-4 and IL-13 in type-2 asthma; 2) therapeutic effects of dupilumab in severe asthma.

\section{PATHOBIOLOGIC ROLES OF IL-4 AND IL-13 IN TYPE 2 ASTHMA}

The main cellular sources of IL-4 and IL-13 include Th2 lymphocytes, $\mathrm{T}$ follicular helper (Tfh) cells and ILC2, and a significant contribution to the production of these two cytokines is also given by eosinophils, basophils, mast cells, 
natural killer cells, and $\mathrm{CD} 8^{+} \mathrm{T}$ lymphocytes (Steinke and Borish, 2001; Vijayanand et al., 2012; Corren, 2013; Maggi et al., 2017; Gowthaman et al., 2019; Komlosi et al., 2022). In regard to the pathobiology of type-2 allergic asthma, IL-4 and IL-13 play relevant roles in airway inflammation and remodeling (Komlosi et al., 2022). Initially released from basophils, IL-4 is essential for differentiation of Th2 cells derived from naïve $\mathrm{CD} 4^{+}$ T lymphocytes (Kaiko et al., 2008; Gandhi et al., 2016). This pathogenic task of IL-4 is further favored by its ability to suppress the immunomodulatory and tolerogenic functions of regulatory T (Treg) cells ( $\mathrm{Tu}$ et al., 2017), which do not allow Th2 cell maturation and clonal expansion in non-allergic subjects (Palomares et al., 2017). Together with IL-13, IL-4 provides the so-called "first signal" that induces IgE production by B cells (Geha et al., 2003; Froidure et al., 2016). IgE class switching also requires a "second signal" for maturation of B lymphocytes, expressed by the B-T cell cognate interaction consisting of an immunological synapse engaging both $\mathrm{T}$ cell receptor (TCR) and CD40/CD40 ligand (CD40L) coupling (Novosad and Krčmová, 2020). Although Th2 and Tfh cells are probably the most relevant cellular sources of IL- 4 and IL13 , these cytokines are also synthesized by ILC2, which thus contribute to stimulate IgE production (Maggi et al., 2017). Upon binding of allergens to adjacent IgE molecules anchored to their high affinity receptors (FceRI) located on the surface of mast cells and basophils, these cells secrete large amounts of IL-4 and IL-13 (Stone et al., 2010; Komlosi et al., 2022), which in turn further amplify the intensity of type-2 airway inflammation.

In addition to T and B lymphocytes, IL-4 and IL-13 act on other cellular targets including both immune/inflammatory and airway structural cells involved in asthma pathophysiology (Figure 1). In particular, IL-13 up-regulates FceRI expression on mast cells, and raises their proliferation (Marone et al., 2019). The most abundant immune cells in the lung are macrophages, which express IL-4/IL-13 receptors whose stimulation drives the polarization towards the M2 macrophage subtype, actively involved in the pathogenesis of severe allergic asthma (Abdelaziz et al., 2020; Becerra-Díaz et al., 2021). IL-4 promotes the attachment of eosinophils to blood vessel walls by enhancing the expression of vascular cell adhesion molecule-1 (VCAM-1), and IL-13 contributes to eosinophil recruitment into the airways by increasing eotaxin synthesis at level of the bronchial epithelium (Rosenberg et al., 2007; Nagata et al., 2020). This tissue is disrupted by IL-13, which down-regulates the expression of claudin-18.1, an important protein component of the intercellular tight junctions that maintain the physical integrity of the airway epithelial barrier (Sweerus et al., 2017). Moreover, IL-4 and IL-13 enhance the expression of histone deacetylases 1 and 9 (HDAC 1 and 9), whose activity is inversely correlated to the integrity of the airway epithelial cell layer (Steelant et al., 2019). Therefore, both IL-4 and IL-13 significantly participate in the induction of bronchial epithelial dysfunction, which is a hallmark of asthma. IL-13 exerts further effects on airway epithelial cells, where this cytokine elicits goblet cell metaplasia and the production of the glycoprotein mucin $5 \mathrm{AC}$ (MUC5AC), which is associated with a more viscous type of mucus (Dickinson et al., 2016). Additionally, in bronchial epithelial cells IL-13 stimulates the expression of the inducible isoform of nitric oxide synthase (iNOS), thereby increasing the airway levels of nitric oxide (Ricciardolo and Silkoff, 2017), a reliable biomarker of type 2 asthma. With regard to airway mesenchymal tissues, IL-13 causes contraction, and proliferation of airway smooth muscle cells (Corren, 2013; Busse et al., 2021). Moreover, IL-13 is a pivotal inducer of bronchial sub-epithelial fibrosis due to fibroblast proliferation and collagen production, and these effects are at least in part mediated by IL-13-dependent activation of transforming growth factor- $\beta 1$ (TGF- $\beta 1$ ) (Lee et al., 2001; Firszt et al., 2014). Such structural changes express the powerful action of IL-13 as a central mediator of airway remodeling in asthma.

Further evidence referring to the very important roles played by IL- 4 and IL-13 in asthma pathobiology ensues from the copious quantities of these cytokines which can be found in bronchial mucosa, induced sputum, bronchoalveolar lavage (BAL) fluid, and peripheral blood from asthmatic patients (Saha et al., 2008; Maes et al., 2012; Corren, 2013). Moreover, exposure of asthmatic subjects to segmental allergen challenges significantly up-regulated airway levels of IL-4/IL-13 mRNAs (Prieto et al., 2000; Saha et al., 2008; Maes et al., 2012; Corren, 2013). Genetic investigations have also detected relevant linkages of IL-13/IL-13 receptor gene polymorphisms with airway hyperresponsiveness and asthma prevalence (Howard et al., 2002). It is thus possible that the genetic control of IL-13 contributes to shape the individual susceptibility to asthma, which is associated with some polymorphisms identified in the RAD50-IL-13 region of chromosome 5q31.1 (Li et al., 2010).

Even if the functional roles of IL-4 and IL-13 are quite overlapping, it is however plausible that these two sister cytokines exert distinct pathobiological actions in asthma. In fact, IL-4 is the key inducer of $\mathrm{CD}^{+}$Th cell commitment towards a Th2 immunophenotype, whilst IL-13 primarily promotes the development of bronchial inflammation and remodeling, thus enhancing airway hyperresponsiveness (Coyle et al., 1995; Grünig et al., 1998; Wills-Karp et al., 1998). Indeed, in comparison to IL4, higher levels of IL-13 can be found in the airways of asthmatic patients, as well as in murine lungs sensitized to allergens (Kotsimbos et al., 1996; Munitz et al., 2008).

IL-4 and IL-13 exert their biologic functions by interacting with specific receptor structures expressed on several target cells (Nelms et al., 1999; Ul-Haq et al., 2016; Chatila, 2004). In particular, only IL-4 binds to type I receptor, a heterodimeric complex in which the IL-4 receptor $\alpha$-subunit (IL-4Ra) is coupled with the $\gamma$ C chain (Figure 1) (Nelms et al., 1999). Both IL-4 and IL-13 can interact with type II receptor, a heterodimer consisting of the IL-4R $\alpha$ component paired with the IL-13 receptor $\alpha 1$ subunit (IL-13Ra1) (McCormick and Heller, 2015).

Binding of IL-4 to type I receptor triggers the activation of Janus kinases 1 (JAK1) and 3 (JAK3), which are linked to the receptor cytoplasmic domains (Wills-Karp and Finkelman, 2008). JAK1 and JAK3 then catalyze the phosphorylation of the tyrosine residues Y500, Y575, Y603, and Y633 localized in the intracellular region of the IL-4Ra subunit, where these phosphorylated amino acids act as a signaling module which recruits key transducing proteins such as insulin receptor 
substrate-2 (IRS-2) and signal transducer and activator of transcription-6 (STAT-6) (Kelly-Welch et al., 2003; Wills-Karp and Finkelman, 2008; Oh et al., 2010; Gour and Wills-Karp, 2015; Harb and Chatila, 2020). IRS-2 proteins bind to the p85 subunit of phosphoinositide-3 kinase (PI3K) and to the adaptor protein growth factor receptor-bound protein 2 (Grb2), which are associated with the PI3K/AKT signaling pathway involved in Th2 cell proliferation (Gour and Wills-Karp, 2015; Pelaia et al., 2017; Harb and Chatila, 2020). Furthermore, as a result of JAK1/ JAK3-mediated tyrosine phosphorylation, STAT-6 undergoes activation, dimerization and nuclear translocation, thus enabling the transcription factor GATA-3 to up-regulate the expression of the genes encoding IL-4, IL-5, and IL-13 (Zeng, 2013; Tindemans et al., 2014; Pelaia et al., 2017; Massey and Suphioglu, 2021).

The type II heterodimeric receptor, composed of IL-4Ra/IL$13 \mathrm{Ra} 1$ subunits and activated by IL-4 and IL-13, is functionally coupled with JAK1/2, tyrosine kinase 2 (Tyk2), and STAT-6, but is not associated with JAK3 and IRS-2 (Andrews et al., 2001; Chiba et al., 2012). IL-13 is also the endogenous ligand of the IL13 receptor $\alpha 2$-chain (IL-13Ra2), which is dissociated from any other receptor protein or signaling network, thereby being responsible for a negative feedback mechanism which inhibits IL-13 functions (Zheng et al., 2008).

\section{THERAPEUTIC EFFECTS OF DUPILUMAB IN SEVERE ASTHMA}

The fully human IgG4 monoclonal antibody dupilumab was realized by Sanofi (Gentilly, France) and Regeneron Pharmaceuticals (Tarrytown, NY, United States) (Shirley, 2017; Santini et al., 2017). Dupilumab specifically binds to IL$4 \mathrm{R} \alpha$, thereby preventing the interaction between IL-4, and the type I receptor complex (Figure 1) (Vatrella et al., 2014). It is also likely that such an IL-4Ra blockade can make it possible for dupilumab to inhibit the recruitment of this subunit, triggered by the interaction of IL-13 with IL-13Ral, and responsible for the assembly of type II receptor (Harb and Chatila, 2020). Therefore, dupilumab behaves as a dual receptor antagonist of IL-4 and IL-13.

Many phase 1 trials were conducted in healthy subjects in order to evaluate the safety and tolerability profile of dupilumab, as well as its pharmacokinetics and immunogenicity (Santini et al., 2017). After subcutaneous administration, dupilumab exhibited a non-linear pharmacokinetics, featured by higher than dose-proportional increments of systemic exposure (Kovalenko et al., 2016; Shirley, 2017). Subsequently to a $600 \mathrm{mg}$ subcutaneous injection, dupilumab displayed an approximate bioavailability of $64 \%$, a total distribution volume of about $4.8 \mathrm{~L}$, and a mean peak concentration at 1 week of $70.1 \mu \mathrm{g} / \mathrm{ml}$ (Vatrella et al., 2014).

The first phase 2a double-blind, randomized, and placebocontrolled trial aimed to assess the therapeutic effects of dupilumab in asthma was carried out in 104 patients (age range: 18-65 years) with moderate-to-severe asthma, characterized by relatively high levels of eosinophils in both blood ( $\geq 300$ cells $/ \mu$ l) and sputum ( $\geq 3 \%$ ) (Wenzel et al., 2013). The inhaled treatment consisted of medium/high doses of inhaled corticosteroids (ICS) and long-acting $\beta_{2}$-adrenergic agonists (LABA), which however did not provide a satisfactory asthma control. Fifty-two patients were randomly treated with weekly subcutaneous administrations of dupilumab $(300 \mathrm{mg})$ for 12 weeks, and 52 subjects were assigned to receive placebo. LABA therapy was interrupted after 4 weeks, whereas ICS treatment was progressively tapered and then suspended between weeks 6 and 9. The primary outcome of this study was to evaluate the impact of dupilumab on asthma exacerbations. Three patients in the dupilumab arm (6\%) and 23 subjects in the placebo group (44\%) experienced a disease exacerbation, respectively; this difference resulted to be statistically significant $(p<0.001)$, and dupilumab reduced the asthma exacerbation rate by $87 \%$. In regard to the secondary endpoints, dupilumab enhanced forced expiratory volume in the first second $\left(\mathrm{FEV}_{1}\right)$ by more than $200 \mathrm{ml}$, and also incremented the morning peak expiratory flow (PEF). Other improvements elicited by dupilumab included a decrease in the score of asthma control questionnaire (ACQ), as well as relevant reductions of nocturnal awakenings, evening and morning symptoms, and inhalation numbers of short-acting bronchodilators used as rescue medication. Furthermore, dupilumab significantly reduced airway and blood levels of several biomarkers of type 2 asthmatic inflammation, including fractional exhaled nitric oxide (FeNO), IgE, CCL26 (eotaxin-3), and TARC (thymus and activation-regulated chemokine). Conversely, dupilumab increased blood eosinophil numbers in 4 patients. With regard to safety and tolerability, in comparison to placebo dupilumab raised the frequency of injection-site reactions, headache, nasopharyngitis, and nausea. Only one patient referred the onset of a cutaneous rush, which promptly recovered after a mild treatment with systemic corticosteroids, and antihistamines. Although this trial provided some valuable information, its study design was not adherent to a real-life setting (Wechsler, 2013; Vatrella et al., 2014). In fact, in daily clinical practice ICS/LABA combinations are not usually suspended during add-on biological treatment of asthma.

The above discrepancy was emended by the study protocol of a larger, subsequent double-blind, randomized, placebo-controlled, dose-ranging, and parallel-group phase $2 \mathrm{~b}$ trial, performed in adult people with persistent asthma, uncontrolled by medium-tohigh doses of ICS/LABA associations, which were not interrupted during add-on treatment with dupilumab (Wenzel et al., 2016). This study was articulated into three phases, consisting of a screening period of 14-21 days, followed by a randomized therapy course of 24 weeks, and by a subsequent posttreatment follow-up lasting 16 weeks. 776 patients were randomly subdivided into five groups, undergoing the following subcutaneous treatments: 1) placebo (158 subjects); 2) dupilumab, $200 \mathrm{mg}$ every 2 weeks (150 subjects); 3) dupilumab, $200 \mathrm{mg}$ every 4 weeks (154 subjects); 4) dupilumab, $300 \mathrm{mg}$ every 2 weeks (157 subjects); 5) dupilumab, $300 \mathrm{mg}$ every 4 weeks (157 subjects). Except for the study arm including patients treated with $200 \mathrm{mg}$ of dupilumab every 4 weeks, in comparison to placebo all the 
other groups experienced significant $\mathrm{FEV}_{1}$ increments, which at the $24^{\text {th }}$ week were comprised between 0.15 and $0.16 \mathrm{~L}$. Additionally, when injected at intervals of 2 weeks, dupilumab significantly decreased the annual numbers of severe asthma exacerbations. All drug dosages also elicited significant and dose-dependent reductions of FeNO, which were quantitatively more relevant when dupilumab was administered every 2 weeks. The effects of dupilumab on asthma exacerbations, pulmonary function and FeNO levels were independent of blood eosinophil counts. Dupilumab was characterized by a good safety and tolerability profile, as shown by the similar distribution across the five study groups of mild adverse events, mainly including reactions at injection site, upper respiratory tract infections, and headache. Temporary elevations of blood eosinophils were detected in some patients with baseline blood eosinophil numbers of at least 300 cells/ $\mu$ l.

The Liberty Asthma Quest trial was a phase 3 double-blind, randomized, placebo-controlled and parallel-group study, which evaluated the efficacy of dupilumab in uncontrolled moderate-tosevere asthma (Castro et al., 2018). In particular, 1902 patients aged at least 12 years were randomly partitioned into four groups, treated for 52 weeks with subcutaneous injections as follows: 1) first loading dosage ( $400 \mathrm{mg}$ ) of dupilumab, followed by a single dose of $200 \mathrm{mg}$ every 2 weeks; 2$)$ matched volume $(1.14 \mathrm{ml})$ of placebo; 3 ) first loading dosage $(600 \mathrm{mg}$ ) of dupilumab, followed by a single dose of $300 \mathrm{mg}$ every 2 weeks; 4) matched volume $(2 \mathrm{ml})$ of placebo. In comparison to placebo arms, patients included in both groups treated with dupilumab experienced a near $50 \%$ decrease in the annualized rate of severe asthma exacerbations. This reduction percentage overcame $65 \%$ in subjects with blood eosinophil counts of 300 or more cells $/ \mu \mathrm{l}$. Both dosages of dupilumab also induced significant $\mathrm{FEV}_{1}$ increases, which resulted to be even greater in patients with at least 300 blood eosinophils/ $\mu \mathrm{L}$. Moreover, dupilumab improved asthma symptom control, as shown by the significant decrements of asthma control questionnaire (ACQ)-5 scores. Furthermore, dupilumab significantly lowered several biomarkers of type 2 asthma, including serum IgE levels, FeNO, as well as the blood concentrations of eotaxin-3, periostin, and TARC. With regard to adverse events, transient increments of blood eosinophils were detected in 52 patients (4.1\%) treated with dupilumab, and in 4 subjects $(0.6 \%)$ who received placebo. A post-hoc analysis of this trial showed that dupilumab decreased severe asthma exacerbations and type 2 inflammatory biomarkers, and also improved asthma control and lung function regardless of the allergic status of treated patients (Corren et al., 2020).

The primary goal of the Liberty Asthma Venture study was to assess the corticosteroid-sparing effect of dupilumab (Rabe et al., 2018). In particular, this phase 3 double-blind, randomized, placebo-controlled trial enrolled 210 patients with oral corticosteroid-dependent severe asthma. These subjects were randomly assigned in a 1:1 ratio to receive for 24 weeks either placebo or an add-on biological therapy with dupilumab, consisting of a first loading dose of $600 \mathrm{mg}$, then followed by a subcutaneous injection of $300 \mathrm{mg}$ every 2 weeks. Study results demonstrated that a $41.9 \%$ reduction of oral glucocorticoid dosage occurred in the placebo arm, whereas a $70.1 \%$ decrease was observed in the dupilumab group. In particular, when compared with the $25 \%$ rate of participants receiving placebo who suspended the oral intake of glucocorticoids, $48 \%$ of patients treated with dupilumab interrupted systemic corticosteroid therapy. In spite of either tapering or discontinuation of oral corticosteroid (OCS) use, in comparison to placebo dupilumab lowered by $59 \%$ the number of severe asthma exacerbations, and also enhanced $\mathrm{FEV}_{1}$ by $220 \mathrm{ml}$. A temporary blood eosinophilia was detected in $14 \%$ of patients treated with dupilumab, and in $1 \%$ of people belonging to the placebo group.

Many patients enrolled in the above trials were also recruited for an open-label extension study named TRAVERSE, which was carried out in 362 hospitals across 27 countries, thus monitoring the effects of dupilumab (first dosage of $600 \mathrm{mg}$, followed by a $300 \mathrm{mg}$ dose administered every 2 weeks) for 96 weeks in 2,282 adults and adolescents with moderate-to-severe asthma (Wechsler et al., 2022). This study confirmed the clinical and functional therapeutic effects of dupilumab, mainly expressed by relevant decreases in asthma symptoms and exacerbations, paralleled by important $\mathrm{FEV}_{1}$ increases. However, the primary aim of TRAVERSE was to investigate the long-term safety of dupilumab. In this regard, dupilumab displayed a sustained profile characterized by good safety and tolerability patterns. The most common adverse events were nasopharyngitis, bronchitis, and erythema at injection site. The most frequent serious adverse events, occurring in very low percentages of patients, included asthma exacerbations and pneumonia. Antidrug antibodies (ADAs) were detected in 157 patients, but did not have any impact on the efficacy and safety of dupilumab. With regard to the biomarkers of type- 2 asthma, dupilumab progressively lowered the levels of both blood eosinophils, and serum total IgE.

The Liberty Asthma Voyage trial was a phase 3, double-blind, randomized, and placebo-controlled study which lasted 52 weeks and evaluated the therapeutic effects of dupilumab in children (age: 6-11 years) with moderate-to-severe asthma (Bacharier et al., 2021). Dupilumab was administered every 2 weeks as add-on biological therapy at doses of either $100 \mathrm{mg}$ (body weight $\leq 30 \mathrm{~kg}$ ) or $200 \mathrm{mg}$ (body weight $>30 \mathrm{~kg}$ ). In particular, 408 asthmatic children were randomly assigned to receive dupilumab or placebo. When compared to placebo, dupilumab significantly reduced the annualized rate of severe asthma exacerbations and also bettered asthma symptom control, as shown by the improvement of the score of Asthma Control Questionnaire 7 Interviewer-Administered (ACQ-7-IA) at week 24. Moreover, add-on treatment with dupilumab had a positive impact on pulmonary function, as demonstrated by the significant increment at week 12 of the percentage of predicted pre-bronchodilator $\mathrm{FEV}_{1}\left(\mathrm{ppFEV}_{1}\right)$. With regard to the occurrence of serious adverse events, no difference was found between the dupilumab group and the placebo arm.

In addition to the above mentioned trials, which are summarized in Table 1, dupilumab has also been recently tested in real-life studies.

A French multi-centre, nationwide, real-life retrospective study was conducted in 64 severe asthmatic patients, with the aim of verifying the effectiveness and safety of dupilumab (Dupin 
TABLE 1 | Dupilumab in severe asthma: summary of the largest clinical trials.

\begin{tabular}{|c|c|c|c|}
\hline Authors & No. Patients & Phase & Main results \\
\hline Wenzel et al., 2013 (ref. 80) & 104 & $2 a$ & Fewer asthma exacerbations, higher $\mathrm{FEV}_{1}$ \\
\hline Wenzel et al., 2016 (ref. 82) & 776 & $2 b$ & Fewer asthma exacerbations, higher $\mathrm{FEV}_{1}$ \\
\hline Castro et al., 2018 (ref. 83) & 1902 & 3 & Fewer asthma exacerbations, better symptom control, higher FEV \\
\hline Rabe et al., 2018 (ref. 85) & 210 & 3 & Lower intake of oral corticosteroids, higher $\mathrm{FEV}_{1}$ \\
\hline Bacharier et al., 2021 (ref. 87) & 408 & 3 & Fewer asthma exacerbations, better symptom control, higher $\mathrm{FEV}_{1}$ \\
\hline Wechsler et al., 2022 (ref. 86) & 2282 & Open-label extension & Long-term safety, fewer asthma exacerbations, higher FEV f $_{1}$ \\
\hline
\end{tabular}

et al., 2020). After 12 months of treatment, the annual number of asthma exacerbations diminished from 4 to 1 , and the score of the asthma control test (ACT) enhanced from 14 (poor symptom control) to 22 (good symptom control). Furthermore, the daily prednisone intake decreased from 20 to $5 \mathrm{mg}$, and the median value of $\mathrm{FEV}_{1}$ increased from 58 to $68 \%$ predicted, corresponding to a median increment of $200 \mathrm{ml}$. Skin reactions at injection-site were the most frequent side effects. Similar clinical and functional benefits were also observed during an investigational period of 1 year, referring to an Italian real-world multicentre study performed in severe asthmatic patients (Campisi et al., 2021).

In a real-life setting, we evaluated the rapidity of dupilumab efficacy in patients with severe asthma and nasal polyposis, who were examined at baseline and after only 4 weeks of treatment (Pelaia et al., 2021). Dupilumab quickly improved symptom control related to both severe asthma and nasal polyposis, as shown by the mean changes regarding the scores of ACT questionnaire (from 12 to 21 ) and sino-nasal outcome test-22 (SNOT-22: from 58 to 19), respectively. Such positive clinical achievements allowed to progressively taper, and then completely eliminate OCS consumption within 4 weeks. Moreover, in the same period we noticed that $\mathrm{FEV}_{1}$, peak expiratory flow (PEF), and forced mid-expiratory flow at $25-75 \%$ of forced vital capacity $\left(\mathrm{FEF}_{25-75}\right)$ increased by more than $200 \mathrm{ml}, 0.6 \mathrm{~L} / \mathrm{s}$, and $0.3 \mathrm{~L} / \mathrm{s}$, respectively. Such a relevant improvement of airflow limitation was associated with a significant reduction of lung hyperinflation, documented by parallel decreases of residual volume (RV: $-690 \mathrm{ml}$ ) and total lung capacity (TLC: $-460 \mathrm{ml}$ ). These last results are very important in consideration of the crucial contribution given by air trapping to the functional abnormalities characterizing severe asthma (Jarjour et al., 2012).

Dupilumab is also very effective as biological therapy of some relevant asthma comorbidities such as nasal polyposis and atopic dermatitis, characterized by type- 2 inflammation (Ricciardolo et al., 2021). Indeed, the two phase 3 multicentre, double-blind,

\section{REFERENCES}

Abdelaziz, M. H., Abdelwahab, S. F., Wan, J., Cai, W., Huixuan, W., Jianjun, C., et al. (2020). Alternatively Activated Macrophages; a Double-Edged Sword in Allergic Asthma. J. Transl Med. 18, 58. doi:10.1186/s12967-020-02251-w

Albrecht, M. (2021). Turning off the Alarm - Targeting Alarmins and Other Epithelial Mediators of Allergic Inflammation with Biologics. Allergol. Select 5, 82-88. doi:10.5414/ALX02194E

Andrews, R., Rosa, L., Daines, M., and Khurana Hershey, G. (2001). Reconstitution of a Functional Human Type II IL-4/IL-13 Receptor in Mouse B Cells: randomized, and placebo-controlled LIBERTY NP SINUS-24 and -52 trials showed that dupilumab significantly ameliorated nasal congestion and obstruction, as well as decreased nasal polyp size and paranasal sinus opacification (Bachert et al., 2019). Moreover, in asthmatic patients with comorbid atopic dermatitis, dupilumab can improve lung function, and reduce the eczema area (Benzecry et al., 2021).

\section{CONCLUDING REMARKS}

The recent advances in phenotypic and endotypic characterization of severe asthma have paved the way for the development of excellent therapeutic tools within the context of biological treatments with monoclonal antibodies. In particular, the IL-4/IL-13 cytokine axis plays a pivotal pathogenic role in type-2 inflammation. Therefore, by effectively blocking at a receptor level the pro-inflammatory mechanisms driven by both IL-4 and IL-13, dupilumab provides valuable benefits for patients with severe type-2 asthma. Dupilumab is especially indicated in the presence of high levels of type-2 biomarkers such as eosinophils (blood cell count $\geq 150$ cells $/ \mu \mathrm{l})$ and FeNO ( $\geq 25 \mathrm{ppb}$ ), eventually associated with OCS-dependence (Moran and Pavord, 2020). Furthermore, dupilumab seems to be very effective in both allergic and non-allergic severe asthma, and exerts its powerful pharmacologic action also when patients complain of asthma comorbidities such as atopic dermatitis and chronic rhinosinusitis with nasal polyps.

\section{AUTHOR CONTRIBUTIONS}

All authors listed have made a substantial, direct, and intellectual contribution to the work and approved it for publication.

Demonstration of Species Specificity. J. Immunol. 166, 1716-1722. doi:10. 4049/jimmunol.166.3.1716

Bacharier, L. B., Maspero, J. F., Katelaris, C. H., Fiocchi, A. G., Gagnon, R., de Mir, I., et al. (2021). Dupilumab in Children with Uncontrolled Moderate-To-Severe Asthma. N. Engl. J. Med. 385, 2230-2240. doi:10.1056/NEJMoa2106567

Bachert, C., Han, J. K., Desrosiers, M., Hellings, P. W., Amin, N., Lee, S. E., et al. (2019). Efficacy and Safety of Dupilumab in Patients with Severe Chronic Rhinosinusitis with Nasal Polyps (LIBERTY NP SINUS-24 and LIBERTY NP SINUS-52): Results from Two Multicentre, Randomised, Double-Blind, Placebo-Controlled, Parallel-Group Phase 3 Trials. Lancet 394, 1638-1650. doi:10.1016/S0140-6736(19)31881-1 
Becerra-Díaz, M., Lerner, A. D., Yu, D. H., Thiboutot, J. P., Liu, M. C., Yarmus, L. B., et al. (2021). Sex Differences in M2 Polarization, Chemokine and IL-4 Receptors in Monocytes and Macrophages from Asthmatics. Cell Immunol 360, 104252. doi:10.1016/j.cellimm.2020.104252

Benzecry, V., Pravettoni, V., Segatto, G., Marzano, A. V., and Ferrucci, S. (2021). Type 2 Inflammation: Atopic Dermatitis, Asthma, and Hypereosinophilia Successfully Treated with Dupilumab. J. Investig. Allergol. Clin. Immunol. 31, 261-263. doi:10.18176/jiaci.0614

Busse, W. W., Kraft, M., Rabe, K. F., Deniz, Y., Rowe, P. J., Ruddy, M., et al. (2021). Understanding the Key Issues in the Treatment of Uncontrolled Persistent Asthma with Type 2 Inflammation. Eur. Respir. J. 58, 2003393. doi:10.1183/ 13993003.03393-2020

Calvén, J., Ax, E., and Rådinger, M. (2020). The Airway Epithelium-A Central Player in Asthma Pathogenesis. Ijms 21, 8907. doi:10.3390/ijms21238907

Campisi, R., Crimi, C., Nolasco, S., Beghè, B., Antonicelli, L., Guarnieri, G., et al. (2021). Real-world Experience with Dupilumab in Severe Asthma: One-Year Data from an Italian Named Patient Program. J. Asthma Allergy 14, 575-583. doi:10.2147/JAA.S312123

Carr, T. F., Zeki, A. A., and Kraft, M. (2018). Eosinophilic and Noneosinophilic Asthma. Am. J. Respir. Crit. Care Med. 197, 22-37. doi:10.1164/rccm.2016112232PP

Castro, M., Corren, J., Pavord, I. D., Maspero, J., Wenzel, S., Rabe, K. F., et al. (2018). Dupilumab Efficacy and Safety in Moderate-To-Severe Uncontrolled Asthma. N. Engl. J. Med. 378, 2486-2496. doi:10.1056/NEJMoa1804092

Chatila, T. A. (2004). Interleukin-4 Receptor Signaling Pathways in Asthma Pathogenesis. Trends Mol. Med. 10, 493-499. doi:10.1016/j.molmed.2004. 08.004

Chiba, Y., Goto, K., and Misawa, M. (2012). Interleukin-13-induced Activation of Signal Transducer and Activator of Transcription 6 Is Mediated by an Activation of Janus Kinase 1 in Cultured Human Bronchial Smooth Muscle Cells. Pharmacol. Rep. 64, 454-458. doi:10.1016/s1734-1140(12)70788-0

Christianson, C. A., Goplen, N. P., Zafar, I., Irvin, C., Good, J. T., Rollins, D. R., et al. (2015). Persistence of Asthma Requires Multiple Feedback Circuits Involving Type 2 Innate Lymphoid Cells and IL-33. J. Allergy Clin. Immunol. 136, 59-e14. doi:10.1016/j.jaci.2014.11.037

Corren, J., Castro, M., O'Riordan, T., Hanania, N. A., Pavord, I. D., Quirce, S., et al. (2020). Dupilumab Efficacy in Patients with Uncontrolled, Moderate-ToSevere Allergic Asthma. J. Allergy Clin. Immunol. Pract. 8, 516-526. doi:10. 1016/j.jaip.2019.08.050

Corren, J. (2013). Role of Interleukin-13 in Asthma. Curr. Allergy Asthma Rep. 13, 415-420. doi:10.1007/s11882-013-0373-9

Coyle, A. J., Le Gros, G., Bertrand, C., Tsuyuki, S., Heusser, C. H., Kopf, M., et al. (1995). Interleukin-4 Is Required for the Induction of Lung Th2 Mucosal Immunity. Am. J. Respir. Cel Mol Biol 13, 54-59. doi:10.1165/ajrcmb.13.1. 7598937

Dickinson, J. D., Alevy, Y., Malvin, N. P., Patel, K. K., Gunsten, S. P., Holtzman, M. J., et al. (2016). IL13 Activates Autophagy to Regulate Secretion in Airway Epithelial Cells. Autophagy 12, 397-409. doi:10.1080/15548627.2015.1056967

Dupin, C., Belhadi, D., Guilleminault, L., Gamez, A. S., Berger, P., De Blay, F., et al. (2020). Effectiveness and Safety of Dupilumab for the Treatment of Severe Asthma in a Real-Life French Multi-centre Adult Cohort. Clin. Exp. Allergy 50, 789-798. doi:10.1111/cea.13614

Firszt, R., Francisco, D., Church, T. D., Thomas, J. M., Ingram, J. L., and Kraft, M. (2014). Interleukin-13 Induces Collagen Type-1 Expression through Matrix Metalloproteinase-2 and Transforming Growth Factor-B1 in Airway Fibroblasts in Asthma. Eur. Respir. J. 43, 464-473. doi:10.1183/09031936. 00068712

Froidure, A., Mouthuy, J., Durham, S. R., Chanez, P., Sibille, Y., and Pilette, C. (2016). Asthma Phenotypes and IgE Responses. Eur. Respir. J. 47, 304-319. doi:10.1183/13993003.01824-2014

Gandhi, N. A., Bennett, B. L., Graham, N. M., Pirozzi, G., Stahl, N., and Yancopoulos, G. D. (2016). Targeting Key Proximal Drivers of Type 2 Inflammation in Disease. Nat. Rev. Drug Discov. 15, 35-50. doi:10.1038/ $\operatorname{nrd} 4624$

Geha, R. S., Jabara, H. H., and Brodeur, S. R. (2003). The Regulation of Immunoglobulin E Class-Switch Recombination. Nat. Rev. Immunol. 3, 721-732. doi:10.1038/nri1181
Gour, N., and Wills-Karp, M. (2015). IL-4 and IL-13 Signaling in Allergic Airway Disease. Cytokine 75, 68-78. doi:10.1016/j.cyto.2015.05.014

Gowthaman, U., Chen, J. S., Zhang, B., Flynn, W. F., Lu, Y., Song, W., et al. (2019). Identification of a T Follicular Helper Cell Subset that Drives Anaphylactic IgE. Science 365 (6456). doi:10.1126/science.aaw6433

Grünig, G., Warnock, M., Wakil, A. E., Venkayya, R., Brombacher, F., Rennick, D. M., et al. (1998). Requirement for IL-13 Independently of IL-4 in Experimental Asthma. Science 282, 2261-2263. doi:10.1126/science.282.5397.2261

Hammad, H., and Lambrecht, B. N. (2021). The Basic Immunology of Asthma. Cell 184, 1469-1485. doi:10.1016/j.cell.2021.02.016

Harb, H., and Chatila, T. A. (2020). Mechanisms of Dupilumab. Clin. Exp. Allergy 50, 5-14. doi:10.1111/cea.13491

Holgate, S. T., Wenzel, S., Postma, D. S., Weiss, S. T., Renz, H., and Sly, P. D. (2015). Asthma. Nat. Rev. Dis. Primers 1, 15025. doi:10.1038/nrdp.2015.25

Hong, H., Liao, S., Chen, F., Yang, Q., and Wang, D. Y. (2020). Role of IL-25, IL-33, and TSLP in Triggering United Airway Diseases toward Type 2 Inflammation. Allergy 75, 2794-2804. doi:10.1111/all.14526

Howard, T. D., Koppelman, G. H., Xu, J., Zheng, S. L., Postma, D. S., Meyers, D. A., et al. (2002). Gene-gene Interaction in Asthma: IL4RA and IL13 in a Dutch Population with Asthma. Am. J. Hum. Genet. 70, 230-236. doi:10. $1086 / 338242$

Jarjour, N. N., Erzurum, S. C., Bleecker, E. R., Calhoun, W. J., Castro, M., Comhair, S. A., et al. (2012). Severe Asthma: Lessons Learned from the National Heart, Lung, and Blood Institute Severe Asthma Research Program. Am. J. Respir. Crit. Care Med. 185, 356-362. doi:10.1164/rccm.201107-1317PP

Kaiko, G. E., Horvat, J. C., Beagley, K. W., and Hansbro, P. M. (2008). Immunological Decision-Making: How Does the Immune System Decide to Mount a Helper T-Cell Response? Immunology 123, 326-338. doi:10.1111/j. 1365-2567.2007.02719.x

Kelly-Welch, A. E., Hanson, E. M., Boothby, M. R., and Keegan, A. D. (2003). Interleukin-4 and Interleukin-13 Signaling Connections Maps. Science 300, 1527-1528. doi:10.1126/science.1085458

Khalaf, K., Paoletti, G., Puggioni, F., Racca, F., De Luca, F., Giorgis, V., et al. (2019). Asthma from Immune Pathogenesis to Precision Medicine. Semin. Immunol. 46, 101294. doi:10.1016/j.smim.2019.101294

Komlosi, Z., van de Veen, W., Kovacs, N., Szűcs, G., Sokolowska, M., O’Mahony, L., et al. (2022). Cellular and Molecular Mechanisms of Allergic Asthma. Mol. Aspects Med. 1. 100995. doi:10.1016/j.mam.2021.100995

Kotsimbos, T. C., Ernst, P., and Hamid, Q. A. (1996). Interleukin-13 and Interleukin-4 Are Coexpressed in Atopic Asthma. Proc. Assoc. Am. Physicians 108, 368-373.

Kovalenko, P., DiCioccio, A. T., Davis, J. D., Li, M., Ardeleanu, M., Graham, N., et al. (2016). Exploratory Population PK Analysis of Dupilumab, a Fully Human Monoclonal Antibody against IL-4Ra, in Atopic Dermatitis Patients and Normal Volunteers. CPT Pharmacometrics Syst. Pharmacol. 5, 617-624. doi:10.1002/psp4.12136

Kuruvilla, M. E., Lee, F. E., and Lee, G. B. (2019). Understanding Asthma Phenotypes, Endotypes, and Mechanisms of Disease. Clin. Rev. Allergy Immunol. 56, 219-233. doi:10.1007/s12016-018-8712-1

Lee, C. G., Homer, R. J., Zhu, Z., Lanone, S., Wang, X., Koteliansky, V., et al. (2001). Interleukin-13 Induces Tissue Fibrosis by Selectively Stimulating and Activating Transforming Growth Factor Beta(1). J. Exp. Med. 194, 809-821. doi:10.1084/jem.194.6.809

Li, X., Howard, T. D., Zheng, S. L., Haselkorn, T., Peters, S. P., Meyers, D. A., et al. (2010). Genome-wide Association Study of Asthma Identifies RAD50-IL13 and HLA-DR/DQ Regions. J. Allergy Clin. Immunol. 125, 328-e11. doi:10.1016/j. jaci.2009.11.018

Maes, T., Joos, G. F., and Brusselle, G. G. (2012). Targeting Interleukin-4 in Asthma: Lost in Translation? Am. J. Respir. Cel Mol Biol 47, 261-270. doi:10. 1165/rcmb.2012-0080TR

Maggi, L., Montaini, G., Mazzoni, A., Rossettini, B., Capone, M., Rossi, M. C., et al. (2017). Human Circulating Group 2 Innate Lymphoid Cells Can Express CD154 and Promote IgE Production. J. Allergy Clin. Immunol. 139, 964-e4. doi:10.1016/j.jaci.2016.06.032

Marone, G., Granata, F., Pucino, V., Pecoraro, A., Heffler, E., Loffredo, S., et al. (2019). The Intriguing Role of Interleukin 13 in the Pathophysiology of Asthma. Front. Pharmacol. 10, 1387. doi:10.3389/fphar.2019.01387 
Massey, O., and Suphioglu, C. (2021). Recent Advances in the Inhibition of the IL-4 Cytokine Pathway for the Treatment of Allergen-Induced Asthma. Int. J. Mol. Sci. 22, 13655. doi:10.3390/ijms222413655

Matucci, A., Bormioli, S., Nencini, F., Maggi, E., and Vultaggio, A. (2021). The Emerging Role of Type 2 Inflammation in Asthma. Expert Rev. Clin. Immunol. 17, 63-71. doi:10.1080/1744666x.2020.1860755

McCormick, S. M., and Heller, N. M. (2015). Commentary: IL-4 and IL13 Receptors and Signaling. Cytokine 75, 38-50. doi:10.1016/j.cyto.2015. 05.023

Moran, A., and Pavord, I. D. (2020). Anti-IL-4/IL-13 for the Treatment of Asthma: the story So Far. Expert Opin. Biol. Ther. 20, 283-294. doi:10.1080/14712598. 2020.1714027

Munitz, A., Brandt, E. B., Mingler, M., Finkelman, F. D., and Rothenberg, M. E. (2008). Distinct Roles for IL-13 and IL-4 via IL-13 Receptor Alphal and the Type II IL-4 Receptor in Asthma Pathogenesis. Proc. Natl. Acad. Sci. U S A. 105, 7240-7245. doi:10.1073/pnas.0802465105

Nagata, M., Nakagome, K., and Soma, T. (2020). Mechanisms of Eosinophilic Inflammation. Asia Pac. Allergy 10, e14. doi:10.5415/apallergy.2020.10.e14

Nelms, K., Keegan, A. D., Zamorano, J., Ryan, J. J., and Paul, W. E. (1999). The IL-4 Receptor: Signaling Mechanisms and Biologic Functions. Annu. Rev. Immunol. 17, 701-738. doi:10.1146/annurev.immunol.17.1.701

Nelson, R. K., Bush, A., Stokes, J., Nair, P., and Akuthota, P. (2020). Eosinophilic Asthma. J. Allergy Clin. Immunol. Pract. 8, 465-473. doi:10.1016/j.jaip.2019.11.024

Novosad, J., and Krčmová, I. (2020). Evolution of Our View on the IgE Molecule Role in Bronchial Asthma and the Clinical Effect of its Modulation by Omalizumab: Where Do We Stand Today? Int. J. Immunopathol Pharmacol. 34, 2058738420942386-15. doi:10.1177/2058738420942386

Oh, C. K., Geba, G. P., and Molfino, N. (2010). Investigational Therapeutics Targeting the IL-4/IL-13/STAT-6 Pathway for the Treatment of Asthma. Eur. Respir. Rev. 19, 46-54. doi:10.1183/09059180.00007609

Palomares, O., Akdis, M., Martín-Fontecha, M., and Akdis, C. A. (2017). Mechanisms of Immune Regulation in Allergic Diseases: the Role of Regulatory T and B Cells. Immunol. Rev. 278, 219-236. doi:10.1111/imr.12555

Papi, A., Brightling, C., Pedersen, S. E., and Reddel, H. K. (2018). Asthma. Lancet 391, 783-800. doi:10.1016/S0140-6736(17)33311-1

Pelaia, C., Crimi, C., Vatrella, A., Tinello, C., Terracciano, R., and Pelaia, G. (2020). Molecular Targets for Biological Therapies of Severe Asthma. Front. Immunol. 11, 603312. doi:10.3389/fimmu.2020.603312

Pelaia, C., Lombardo, N., Busceti, M. T., Piazzetta, G., Crimi, C., Calabrese, C., et al. (2021). Short-term Evaluation of Dupilumab Effects in Patients with Severe Asthma and Nasal Polyposis. J. Asthma Allergy 14, 1165-1172. doi:10.2147/ JAA.S328988

Pelaia, C., Paoletti, G., Puggioni, F., Racca, F., Pelaia, G., Canonica, G. W., et al. (2019). Interleukin-5 in the Pathophysiology of Severe Asthma. Front. Physiol. 10, 1514. doi:10.3389/fphys.2019.01514

Pelaia, C., Pelaia, G., Crimi, C., Longhini, F., Lombardo, N., Savino, R., et al. (2022). Biologics in Severe Asthma. Minerva Med. Epub ahead of print. doi:10.23736/ S0026-4806.21.07296-7

Pelaia, C., Vatrella, A., Gallelli, L., Terracciano, R., Navalesi, P., Maselli, R., et al. (2017). Dupilumab for the Treatment of Asthma. Expert Opin. Biol. Ther. 17, 1565-1572. doi:10.1080/14712598.2017.1387245

Porsbjerg, C. M., Sverrild, A., Lloyd, C. M., Menzies-Gow, A. N., and Bel, E. H. (2020). Anti-alarmins in Asthma: Targeting the Airway Epithelium with Next-Generation Biologics. Eur. Respir. J. 56, 2000260. doi:10.1183/13993003.00260-2020

Prieto, J., Lensmar, C., Roquet, A., van der Ploeg, I., Gigliotti, D., Eklund, A., et al. (2000). Increased Interleukin-13 mRNA Expression in Bronchoalveolar Lavage Cells of Atopic Patients with Mild Asthma after Repeated Low-Dose Allergen Provocations. Respir. Med. 94, 806-14. doi:10.1053/rmed.2000.0826

Rabe, K. F., Nair, P., Brusselle, G., Maspero, J. F., Castro, M., Sher, L., et al. (2018). Efficacy and Safety of Dupilumab in Glucocorticoid-dependent Severe Asthma. N. Engl. J. Med. 378, 2475-2485. doi:10.1056/NEJMoa1804093

Ricciardolo, F. L. M., Bertolini, F., and Carriero, V. (2021). The Role of Dupilumab in Severe Asthma. Biomedicines 9, 1096. doi:10.3390/biomedicines9091096

Ricciardolo, F. L. M., and Silkoff, P. E. (2017). Perspectives on Exhaled Nitric Oxide. J. Breath Res. 11, 047104. doi:10.1088/1752-7163/aa7f0e

Rodriguez-Rodriguez, N., Gogoi, M., and McKenzie, A. N. J. (2021). Group 2 Innate Lymphoid Cells: Team Players in Regulating Asthma. Annu. Rev. Immunol. 39, 167-198. doi:10.1146/annurev-immunol-110119-091711
Rosenberg, H. F., Phipps, S., and Foster, P. S. (2007). Eosinophil Trafficking in Allergy and Asthma. J. Allergy Clin. Immunol. 119, 1303-2. doi:10.1016/j.jaci. 2007.03.048

Saha, S. K., Berry, M. A., Parker, D., Siddiqui, S., Morgan, A., May, R., et al. (2008). Increased Sputum and Bronchial Biopsy IL-13 Expression in Severe Asthma. J. Allergy Clin. Immunol. 121, 685-91. doi:10.1016/j.jaci.2008.01.005

Santini, G., Mores, N., Malerba, M., Mondino, C., Anzivino, R., Macis, G., et al. (2017). Dupilumab for the Treatment of Asthma. Expert Opin. Investig. Drugs 26, 357-366. doi:10.1080/13543784.2017.1282458

Shirley, M. (2017). Dupilumab: First Global Approval. Drugs 77, 1115-1121. doi:10.1007/s40265-017-0768-3

Steelant, B., Wawrzyniak, P., Martens, K., Jonckheere, A. C., Pugin, B., Schrijvers, R., et al. (2019). Blocking Histone Deacetylase Activity as a Novel Target for Epithelial Barrier Defects in Patients with Allergic Rhinitis. J. Allergy Clin. Immunol. 144, 1242-e7. e1247. doi:10.1016/j.jaci.2019.04.027

Steinke, J. W., and Borish, L. (2001). Th2 Cytokines and Asthma. Interleukin-4: its Role in the Pathogenesis of Asthma, and Targeting it for Asthma Treatment with Interleukin-4 Receptor Antagonists. Respir. Res. 2, 66-70. doi:10.1186/rr40

Stern, J., Pier, J., and Litonjua, A. A. (2020). Asthma Epidemiology and Risk Factors. Semin. Immunopathol 42, 5-15. doi:10.1007/s00281-020-00785-1

Stone, K. D., Prussin, C., and Metcalfe, D. D. (2010). IgE, Mast Cells, Basophils, and Eosinophils. J. Allergy Clin. Immunol. 125, S73-80. doi:10.1016/j.jaci.2009.11.017

Suraya, R., Nagano, T., Katsurada, M., Sekiya, R., Kobayashi, K., and Nishimura, Y. (2021). Molecular Mechanism of Asthma and its Novel Molecular Target Therapeutic Agent. Respir. Investig. 59, 291-301. doi:10.1016/j.resinv.2020. 12.007

Sweerus, K., Lachowicz-Scroggins, M., Gordon, E., LaFemina, M., Huang, X., Parikh, M., et al. (2017). Claudin-18 Deficiency Is Associated with Airway Epithelial Barrier Dysfunction and Asthma. J. Allergy Clin. Immunol. 139, 72-e1. doi:10.1016/j.jaci.2016.02.035

Thomsen, S. F. (2015). The Contribution of Twin Studies to the Understanding of the Aetiology of Asthma and Atopic Diseases. Eur. Clin. Respir. J. 2, 27803. doi:10.3402/ecrj.v2.27803

Tindemans, I., Serafini, N., Di Santo, J. P., and Hendriks, R. W. (2014). GATA-3 Function in Innate and Adaptive Immunity. Immunity 41, 191-206. doi:10. 1016/j.immuni.2014.06.006

Tliba, O., and Panettieri, R. A., Jr (2019). Paucigranulocytic Asthma: Uncoupling of Airway Obstruction from Inflammation. J. Allergy Clin. Immunol. 143, 1287-1294. doi:10.1016/j.jaci.2018.06.008

Tu, L., Chen, J., Zhang, H., and Duan, L. (2017). Interleukin-4 Inhibits Regulatory T Cell Differentiation through Regulating CD103+ Dendritic Cells. Front. Immunol. 8, 214. doi:10.3389/fimmu.2017.00214

Ul-Haq, Z., Naz, S., and Mesaik, M. A. (2016). Interleukin-4 Receptor Signaling and its Binding Mechanism: a Therapeutic Insight from Inhibitors Tool Box. Cytokine Growth Factor. Rev. 32, 3-15. doi:10.1016/j.cytogfr.2016.04.002

Vatrella, A., Fabozzi, I., Calabrese, C., Maselli, R., and Pelaia, G. (2014). Dupilumab: a Novel Treatment for Asthma. J. Asthma Allergy 7, 123-30. doi:10.2147/JAA.S52387

Vijayanand, P., Seumois, G., Simpson, L. J., Abdul-Wajid, S., Baumjohann, D., Panduro, M., et al. (2012). Interleukin-4 Production by Follicular Helper T Cells Requires the Conserved Il4 Enhancer Hypersensitivity Site V. Immunity 36, 175-87. doi:10.1016/j.immuni.2011.12.014

Wechsler, M. E., Ford, L. B., Maspero, J. F., Pavord, I. D., Papi, A., Bourdin, A., et al. (2022). Long-term Safety and Efficacy of Dupilumab in Patients with ModerateTo-Severe Asthma (TRAVERSE): an Open-Label Extension Study. Lancet Respir. Med. 10, 11-25. doi:10.1016/S2213-2600(21)00322-2

Wechsler, M. E. (2013). Inhibiting Interleukin-4 and Interleukin-13 in DifficultTo-Control Asthma. N. Engl. J. Med. 368, 2511-3. doi:10.1056/NEJMe1305426

Wenzel, S., Castro, M., Corren, J., Maspero, J., Wang, L., Zhang, B., et al. (2016). Dupilumab Efficacy and Safety in Adults with Uncontrolled Persistent Asthma Despite Use of Medium-To-High-Dose Inhaled Corticosteroids Plus a LongActing $\beta 2$ Agonist: a Randomised Double-Blind Placebo-Controlled Pivotal Phase 2b Dose-Ranging Trial. Lancet 388, 31-44. doi:10.1016/S0140-6736(16) 30307-5

Wenzel, S., Ford, L., Pearlman, D., Spector, S., Sher, L., Skobieranda, F., et al. (2013). Dupilumab in Persistent Asthma with Elevated Eosinophil Levels. N. Engl. J. Med. 368, 2455-66. doi:10.1056/NEJMoa1304048 
Wenzel, S. E. (2021). Severe Adult Asthmas: Integrating Clinical Features, Biology, and Therapeutics to Improve Outcomes. Am. J. Respir. Crit. Care Med. 203, 809-821. doi:10.1164/rccm.202009$3631 \mathrm{CI}$

Wills-Karp, M., and Finkelman, F. D. (2008). Untangling the Complex Web of IL4- and IL-13-mediated Signaling Pathways. Sci. Signal. 1, pe55. doi:10.1126/ scisignal.1.51.pe55

Wills-Karp, M., Luyimbazi, J., Xu, X., Schofield, B., Neben, T. Y., Karp, C. L., et al. (1998). Interleukin-13: central Mediator of Allergic Asthma. Science 282, 2258-61. doi:10.1126/science.282.5397.2258

Zeng, W. P. (2013). 'All Things Considered': Transcriptional Regulation of T Helper Type 2 Cell Differentiation from Precursor to Effector Activation. Immunology 140, 31-8. doi:10.1111/imm.12121

Zheng, T., Liu, W., Oh, S. Y., Zhu, Z., Hu, B., Homer, R. J., et al. (2008). IL-13 Receptor Alpha2 Selectively Inhibits IL-13-induced Responses in the Murine Lung. J. Immunol. 180, 522-9. doi:10.4049/jimmunol.180. 1.522
Conflict of Interest: The authors declare that the research was conducted in the absence of any commercial or financial relationships that could be construed as a potential conflict of interest.

Publisher's Note: All claims expressed in this article are solely those of the authors and do not necessarily represent those of their affiliated organizations, or those of the publisher, the editors and the reviewers. Any product that may be evaluated in this article, or claim that may be made by its manufacturer, is not guaranteed or endorsed by the publisher.

Copyright (c) 2022 Pelaia, Heffler, Crimi, Maglio, Vatrella, Pelaia and Canonica. This is an open-access article distributed under the terms of the Creative Commons Attribution License (CC BY). The use, distribution or reproduction in other forums is permitted, provided the original author(s) and the copyright owner(s) are credited and that the original publication in this journal is cited, in accordance with accepted academic practice. No use, distribution or reproduction is permitted which does not comply with these terms. 